\title{
Dépôt de films minces par ablation laser, le potentiel industriel
}

\author{
C. Belouet, D. Chambonnet, D. Keller et C. Villard*
}

Alcatel Alsthom Recherche, route de Nozay, 91460 Marcoussis, France

* EPM-Matformag, 25 avenue des Martyrs, 38042 Grenoble cedex 9, France

\begin{abstract}
Résumé : cet article présente brièvement le potentiel de la technique de dépôt de films minces par ablation d'une cible au moyen d'un faisceau laser pulsé. Il donne une liste d'utilisations, le plus souvent en laboratoire, de cette technique par l'industrie. Quełques exemples sont donnés à titre d'illustration : films de carbone DLC, de cuprate supraconducteur $\mathrm{YBa}_{2} \mathrm{Cu}_{3} \mathrm{O}_{7 . \delta}$, d'alliage métallique à base Ni et de métallisation AuSn. Les étapes à sumonter avant d'envisager de faire de ce nouvel outil un moyen industriel sont discutées.
\end{abstract}

\section{Introduction}

Il existe aujourd'hui un grand nombre de techniques de dépôt pour réaliser les films minces nécessaires à l'électronique, à l'optoélectronique, à l'optique, voire à la métallurgie, tableau 1.

Tableau 1 : exemples de techniques de dépôt de films minces classées en quatre grandes familles

\begin{tabular}{|c|c|}
\hline Famille & Exemples \\
\hline 1- dépôt chimique en solution & $\begin{array}{l}\text { épitaxie en phase liquide (LPE) } \\
\text { dépôt électrochimique, } \\
\text { sol-gel, ... }\end{array}$ \\
\hline $\begin{array}{l}\text { 2- dépôt chimique en phase vapeur } \\
\text { (CVD) }\end{array}$ & $\begin{array}{l}\text { MOCVD, } \\
\text { plasma CVD, plasma ECR, } \\
\text { épitaxje en phase vapeur (VPE) ... }\end{array}$ \\
\hline $\begin{array}{l}\text { 3- dépôt physique en phase vapeur } \\
\text { (PVD) }\end{array}$ & $\begin{array}{l}\text { pulvérisation } R F \text {, magnétron, ... } \\
\text { évaporation, ... }\end{array}$ \\
\hline 4- dépôt sous vide & $\begin{array}{l}\text { pulvérisation par faisceau d'ions, } \\
\text { épitaxie par jet moléculaire (MBE) }\end{array}$ \\
\hline
\end{tabular}

CVD : chemical vapour deposition ; PVD : physical vapour deposition ; LPE : liquid phase epitaxy ; MOCVD : metal organic CVD ; ECR : electron cyclotron resonance ; VPE : vapour phase epitaxy ; MBE : molecular beam epitaxy.

Un procédé de dépôt industriel doit satisfaire certaines conditions techniques minimales, dont : une vitesse effective de dépôt élevée et une homogénéité de la composition et des caractéristiques appropriées du film sur de grandes surfaces (on envisage par exemple le passage au dépôt sur plaques de diamètre 30 voire $40 \mathrm{~cm}$ en technologie silicium au cours de la prochaine décennie).

Pour la plupart des matériaux connus, il existe déjà plusieurs méthodes de dépôt souvent concurrentes, techniquement appropriées et économiquement viables. Dans certains secteurs, des méthodes se sont par ailleurs imposées sinon définitivement, du moins pour une longue période, telles les techniques MOCVD et MBE pour les composés III-V. C'est dans ce contexte, où l'industrie dispose d'une panoplie de moyens de dépôt bien établis, que s'est développée la technique de dépôt par ablation d'une cible du matériau à déposer au moyen d'un laser pulsé. Cette technique, nommée par la suite PLD (sigle anglais pour puised laser-assisted deposition), proposée dans les années 60 par H.M.Smith et A.F.Turner [1], a été développée dès les années 70 en ex-URSS, et a connu une croissance rapide en Occident, au Japon et en Chine à la fin des années 80 , stimulée par la découverte des cuprates supraconducteurs à haute température critique (SHTc) et avec $\mathrm{YBa}_{2} \mathrm{Cu}_{3} \mathrm{O}_{7-\delta}(\mathrm{YBCO})$ en particulier [2-3]. Ces travaux sur les SHTc ont 
mis en évidence deux atouts majeurs de la PLD, largement mis à profit par la suite : le transfert quasi-congruent de la composition du matériau cible et la possibilité de travailler sous des pressions relativement élevées. Depuis lors, la technique a été utilisée en laboratoire pour le dépôt d'une gamme étendue de matériaux et plus particulièrement les composés d'oxydes complexes. Cet article, consacré au potentiel industriel de la technique PLD, présente successivement, après un bref rappel du principe de la technique et des processus mis en jeu, ses secteurs d'applications explorés dans les laboratoires industriels, ses atouts et ses limitations avec leurs solutions possibles. Plusieurs actions nécessaires pour faire passer à terme cette technique d'un outil de laboratoire très performant à un moyen de production sont évoquées.

\section{La technique PLD}

\subsection{Le principe}

La technique consiste à irradier sous une forte densité de puissance la surface du matériau à déposer (cible) par un faisceau laser pulsé de courte longueur d'onde. L'énergie optique, absorbée sur une faible profondeur, provoque l'émission de matière -électrons, ions, radicaux et molécules- suivant un panache en forme de cône peu ouvert et dont l'axe se situe dans une direction proche de la normale à la surface de la cible. Simultanément, des gouttelettes, dont la taille, micrométrique en général, et le nombre dépendent beaucoup du matériau cible et des conditions de l'ablation, sont émises par la surface fondue. Ce processus parasite constitue aujourd'hui une limitation importante au développement industriel de la technique PLD. Le principe de cette technique, objet de nombreux articles de revue [4-8], est illustré dans la figure 1 .

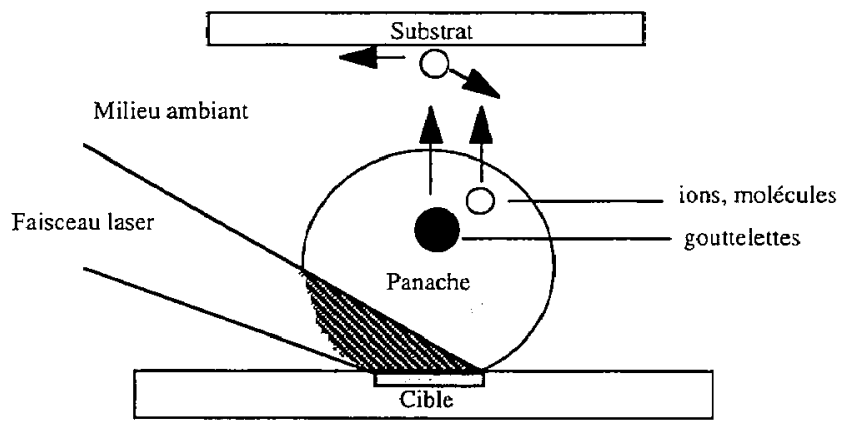

Figure 1. Schéma de principe de la technique PLD

Ce procédé occupe une niche étroite parmi l'ensemble des techniques de traitement de la matière par irradiation au moyen d'un laser pulsé. Il est caractérisé par une densité de puissance $P$, de $10^{6}$ à $10^{8} \mathrm{~W} / \mathrm{cm}^{2}$, une durée d'impulsion $\tau$ de 1 à $30 \mathrm{~ns}$ et une longueur d'onde située dans l'ultra violet. Le tableau 2 présente quelques uns des lasers les plus fréquemment utilisés en laboratoire.

Tableau 2 : caractéristiques des impuisions des lasers utilisés en technique PLD

\begin{tabular}{lll}
\hline caractéristiques de l'impulsion & Nd:YAG & Excimère \\
\hline longueur d'onde, $\lambda(\mathrm{nm})$ & 266,351 & $157^{*}, 193,248,308,351$ \\
énergie $(\mathrm{mJ})$ & $100-300$ & $100-800$ \\
dimension du faisceau (mm) & $9-12^{(1)}$ & $10 \times 30^{(2)}$ \\
durée (ns) & $4-12$ & $20-30$ \\
fréquence $(\mathrm{Hz})$ & $1-100$ & $1-500$ \\
faisceau circulaire ${ }^{(1)}$ et rectangulaire ${ }^{(2)}$ & & \\
* matériel récemment proposé sur le marché & \\
\hline
\end{tabular}

Une description phénoménologique de la technique PLD fait intervenir plusieurs processus fondamentaux, dont : 1 . le transfert de l'énergie optique incidente à la matière 
[9], 2. l'émission de matière, espèces moléculaires et gouttelettes [10-15], 3. la propagation de l'interface solide-liquide à partir de la surface de la cible, décrite par les lois de la thermique classique pour les durées d'impulsion usuelles, la stabilité de la surface liquide [16] et la recristallisation de la zone fondue, 4. la dynamique de la propagation du panache vers le substrat et l'absorption des photons incidents par la matière du panache [17] et 5 . la croissance du film sur le substrat par la collecte des espèces éjectées. De nombreux travaux ont été consacrés à la compréhension de ces différents processus, dont certains sont encore mal décrits : par exemple, l'énergie élevée des espèces moléculaires émises, qui peut atteindre des dizaines d'électrons-Volts, valeur très supérieure à celle attendue d'une émission thermique classique.

\subsection{Le potentiel}

Le potentiel de la technique est fondé sur un ensemble de propriétés résumées ci-après : 1. le transfert congruent (cations) de la composition de la cible, 2. le dépôt applicable à un large spectre de matériaux, 3. l'utilisation possible de précurseurs sans hydrogène et/ou carbone, 4. le domaine de pression de dépôt très étendu avec Ies possibilités de créer un plasma, indépendant du transfert de matière par ablation, entre la cible et le substrat [18], de ralentir l'énergie cinétique des espèces moléculaires du panache, de générer des réactions chimiques moléculaires [19] ou la nucléation d'amas par collisions entre les molécules du milieu ambiant et du panache [20] et enfin 5. I'utilisation séquentielle de plusieurs cibles avec les possibilités de réaliser des couches à gradient de composition,des multicouches et des structures artificielles (à base d'oxydes complexes par exemple [21]).

Ces propriétés ont été rapidement identifiées et mises à profit par de nombreux laboratoires universitaires et industriels, tableau 3.

Tableau 3 : exemples de composés, classés par famille chirnique, déposés en films minces par la technique PLD en milieu industriel (ou à des fins d'applications). Les champs d'applications, cités dans les références, sont donnés à titre indicatif.

\begin{tabular}{|c|c|c|}
\hline FAMILLE / Propriétés & COMPOSE /Substrat & APPLICATIONS /Motivation \\
\hline $\begin{array}{l}\text { 1- CARBURES } \\
\text { émission de champ } \\
\text { résistivité, optique } \\
\text { matériau H.T. }\end{array}$ & $\begin{array}{l}\text { DLC }\left(\mathrm{sp}^{3}\right) \\
\mathrm{DLC}\left(\mathrm{sp}^{3}\right) \\
\mathrm{TiC} / \mathrm{Si}\end{array}$ & $\begin{array}{l}\text { écrans plats [25 ] } \\
\text { électronique, optique [26] } \\
\text { protection, tribologie (bt) }[27]^{(1)}\end{array}$ \\
\hline 2- NITRURES & $\begin{array}{l}\text { BN (cubique) } \\
\text { TiN }\end{array}$ & $\begin{array}{l}\text { électronique (bt) [28] } \\
\text { électronique [29] }\end{array}$ \\
\hline $\begin{array}{l}\text { 3- OXYDES } \\
\text { ferroélectriques }\end{array}$ & 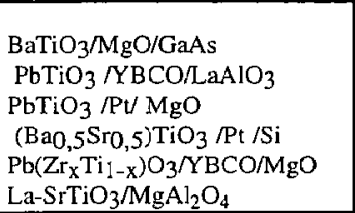 & $\begin{array}{l}\text { dispositifs O/E (bt) [30] } \\
\text { détection IR (st) [31] } \\
\text { NVM's, détection IR,(bt, st, ht) [32] } \\
\text { DRAM's (bt, hp, st) [33] } \\
\text { DRAM's (bt, st) [34] } \\
\text { capteurs, DRAM's[35] }\end{array}$ \\
\hline $\begin{array}{l}\text { magnéto-optiques } \\
\text { magnétiques } \\
\text { piézo-électriques, E/O } \\
\text { supraconductrices HTc }\end{array}$ & $\begin{array}{l}\text { YIG : BIG multicouche } \\
\mathrm{Lal}-\mathrm{xSrXMnO}_{3} \\
\mathrm{LiNbO}_{3}-\mathrm{LiTaO}_{3} \\
\mathrm{YBa}_{2} \mathrm{Cu}_{3} \mathrm{O}_{7-\delta} \\
\mathrm{HgBa}_{2} \mathrm{Ca}_{1} \mathrm{Cu}_{2} \mathrm{O}_{4}\end{array}$ & $\begin{array}{l}\text { mémoires magnétoopt.(bt, st) [36] } \\
\text { magnéto-résistance géante [37] } \\
\text { SAW, optique intégrée (bt,st) [38] } \\
\text { composants hyperfréquences [39] } \\
\text { ibidem [40] }\end{array}$ \\
\hline $\begin{array}{l}\text { 4-METAUX/ ALLIAGES } \\
\text { magnétiques } \\
\text { conductrices } \\
\text { thermomécaniques }\end{array}$ & $\begin{array}{l}\text { nanostructures } \mathrm{CoCu} / \mathrm{Si} \\
\mathrm{Nd}_{2} \mathrm{Fe}_{14} \mathrm{~B} \\
\text { eutectique AuSn }(80-20 \% \text { en } \\
\text { masse } \\
\mathrm{NiAl} \\
\mathrm{W} / \mathrm{GaAs} \\
\mathrm{NiCrAlCoY}\end{array}$ & $\begin{array}{l}\text { capteurs magnétiques [41] } \\
\text { aimant permanent, O/E [42] } \\
\text { hybrid. flip-chip, O/E [cet article] } \\
\text { (st) } \\
\text { électronique [43] }(1) \\
\text { électronique, barrière [44] } \\
\text { aubes de TAG[st, grad.][cet article] }\end{array}$ \\
\hline $\begin{array}{l}\text { 5- DIVERS } \\
\text { polymères } \\
\text { semi-conducteur /verre }\end{array}$ & $\begin{array}{l}\text { PTFE } \\
\text { CdTe } \mu \text { cristallites }\end{array}$ & $\begin{array}{l}\text { dépôt sans solvant [45] } \\
\text { dispositifs optiques [46] }\end{array}$ \\
\hline
\end{tabular}

(1) finalité : application (hors industrie) ; bt : procédé basse température, ht : haute pression, st : dépôt stoechiométrique, grad : dépôt de couche à gradient de composition, DRAM : dynamic random access 
memory, HT : (matériau) haute température : NVM : non volatile memory, O/E : optoélectronique, SAW : surface acoustic wave. TAG : turbine à gaz.

Les exemples ci-dessous d'utilisation de la technique PLD dans notre laboratoire illustrent son potentiel.

\subsection{1 exemples d'applications particuliers}

2.2.1.1 dépôt de carbone DLC . Le contrôle de la structure des films de carbone adamantin (DLC, diamond-like carbon), caractérisée par le rapport , $r$, entre le nombre de liaisons de type diamant - $\mathrm{sp}^{3}$ - et le nombre de liaisons de type graphite $\mathrm{sp}^{2}\left(r=\mathrm{sp}^{3}\right)$ $\mathrm{sp}^{2}$ ), est directement lié à celui de l'énergie des espèces moléculaires incidentes. La structure en majorité $\mathrm{sp}^{3}$ est obtenue au-dessus d'un seuil d'énergie de ces espèces de l'ordre de $120 \mathrm{eV}$ [22]. En dessous de ce seuil, la variété $\mathrm{sp}^{2}$ prédomine. Certaines caractéristiques physiques de ces deux variétés diffèrent dans de larges proportions : propriétés optiques, conductibilité thermique, $\mathrm{k}$ (des valeurs de $\mathrm{k}=1000 \mathrm{~W} \cdot \mathrm{K}^{-1} \cdot \mathrm{m}^{-1}$ ont été citées pour la variété $\mathrm{sp}^{3}$ [23]), contraintes internes, etc... L'épaisseur des films de la variété $\mathrm{sp}^{3}$ est limitée à des valeur de l'ordre de $1 \mu \mathrm{m}$, seuil typique au-delà duquel le dépôt se sépare de son support en raison de contraintes internes très élevées [24]. Des structures multicouches, constituées de séquences $\mathrm{sp}^{3}-\mathrm{sp}^{2}$, permettent d'atteindre des épaisseurs très supérieures sans fissuration ou pelage des dépôts. Ces structures sont obtenues en PLD par simple ajustement de la pression dans l'enceinte de dépôt, la variêté $\mathrm{sp}^{3}$ étant obtenue à basse pression $\left(<10^{-5} \mathrm{mbar}\right)$ et la variété $\mathrm{sp}^{2}$, sous une pression (argon par exemple) de l'ordre de 0,1 mbar.

2.2.1.2 dépôt de métallisation AuSn pour hybridation par la technique "flip-chip". Les bossages à base d'alliage AuSn à la composition eutectique (80-20\% en masse) sont utilisés en optoélectronique sous forme de mesas de hauteur inférieure à $10 \mu \mathrm{m}$ pour l'hybridation de sources laser sur support en silicium par exemple. Les mesas, localisées par photolithographie et isolées par la technique du 'lift-off', sont classiquemeent déposées par évaporation sous vide ou par voie électrochimique. Elles sont constituées d'un empilement de couches Au, Sn, dont les épaisseurs respectives sont ajustées pour réaliser la composition eutectique. La maîtrise de la fusion d'une telle structure est relativement critique, en raison notamment du risque de formation de phases solides solubles à des températures bien supérieures à celle de l'eutectique $\left(278^{\circ} \mathrm{C}\right)$ [47]. La technique PLD permet de préparer directement l'alliage à sa composition eutectique par pulvérisation d'une cible de AuSn, moyennant quelques précautions. Aux fluences ordinaires de quelques $\mathrm{J} / \mathrm{cm}^{2}(\lambda=248 \mathrm{~nm})$, et sous faible pression, le dépôt est très appauvri en étain et le taux de dépôt par tir est très faible, figure 2 . Ce phénomène est dû à un processus de re-pulvérisation, particulièrement efficace en ce qui concerne l'étain. A plus haute pression, les espèces du panaches sont en partie ralenties par collision avec les molécules du milieu ambiant [48] et on observe simultanément une remontée du taux de dépôt et une forte réduction de l'écart entre les compositions élémentaires de la cible et du dépôt. Le procédé est optimisé en ajustant le couple pression - composition de la cible, qui est associé à un taux de dépôt acceptable et à une faible résistivité électrique de l'alliage déposé.

2.2.1.3 les couches à gradient de composition. Ces structures sont couramment utilisées en technologie semi-conducteur pour I- réaliser des super-réseaux contraints ou plus simplement, pour 2- passer continûment du substrat au film actif lorsque ces matériaux ont une même structure et des paramètres cristallins très voisins. La technique PLD, séquentielle dans son principe même, est particulièrement bien adaptée à ce problème. Deux exemples d'utilisation dans notre laboratoire illustrent ce potentiel : le dépôt d'un revêtement anticorrosion à base de $\mathrm{NiCrAlY}$ pour les aubes de turbines à gaz monocristallines (alliage base Ni, AM1 [49]) et la réalisation d'une couche tampon pour le dépôt de $\mathrm{YBa}_{2} \mathrm{Cu}_{3} \mathrm{O}_{7.5}$ sur un substrat modèle, $\mathrm{SrTiO}_{3}$.

Les problèmes à résoudre dans le premier cas concernent entre autres la continuité de la composition à l'interface substrat-film (critère de cohésion) et le contrôle de la composition du revêtement protecteur (critère de protection). Une couche tampon à gradient de composition, qui permet de passer régulièrement de la composition de 


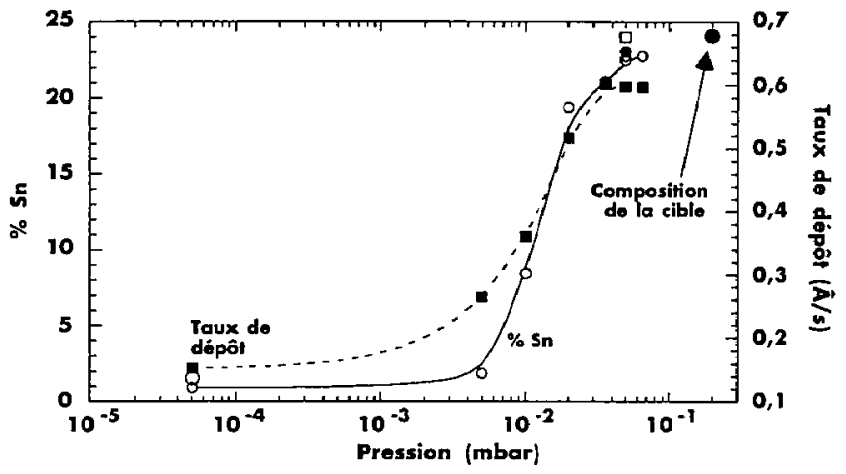

Figure 2 : composition en étain dans le film (masse \%) et taux de dépôt en fonction de la pression dans l'enceinte de dépôt.

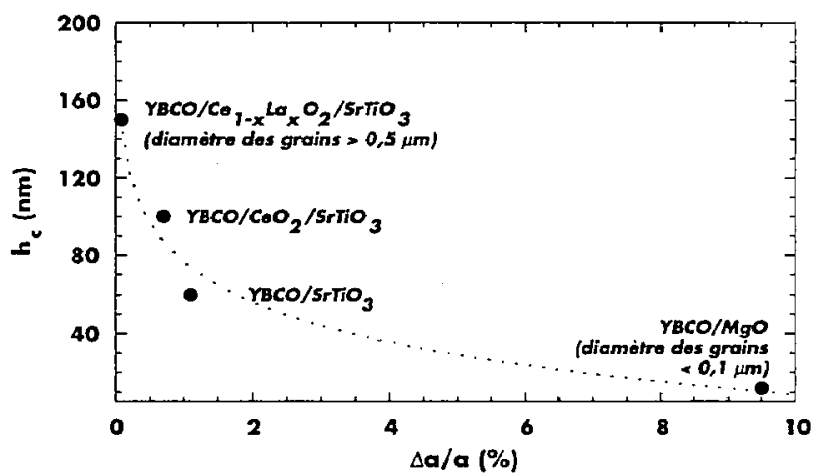

Figure 3 : hauteur critique $h_{c}$ en fonction de l'écart relatif de paramètre cristallin entre YBCO et le film sous-jacent (à l'interface avec YBCO)

l'alliage AM1 à celle du revêtement, est une solution à ces problèmes. Cette couche est obtenue au moyen de deux cibles - à la composition de l'alliage AMl et du revêtementexposées au faisceau laser suivant une séquence programmée et contrôlée par ordinateur.

L'interêt de la technique PLD est ici le transfert quasi-exact de la composition des cibles.

Le problème à résoudre dans le second cas est celui de la réduction des effets granulaires dans les films supraconducteurs destinés à la fabrication de composants passifs en hyperfréquences [50]. Une étude de ces effets a montré que l'amélioration des performances de ces dispositifs était directement liée à l'obtention d'une texture cristalline à gros grains, parfaitement contrôlée : axe $\mathrm{c}$ normal à la couche et axes $a, b$ parallèles aux directions $[100]$ et $[100]$ du substrat $\left(\{001\} \mathrm{LaAlO}_{3}\right.$ par exemple). La réalisation d'une couche à gradient de composition est une réponse pertinente à ce problème comme l'a démontré l'étude d'une structure modèle $\mathrm{SrTiO}_{3} / \mathrm{CeO}_{2}-\mathrm{La} / \mathrm{YBa}_{2} \mathrm{Cu}_{3} \mathrm{O}_{7-\delta}$, dans laquelle la couche intermédiaire de cérine dopée au lanthane permet de passer continûment du paramètre de $\mathrm{SrTiO}_{3}$ à celui du film $\mathrm{YBa}_{2} \mathrm{Cu}_{3} \mathrm{O}_{7-\delta}$ à la température du dépôt, par variation du taux de dopage. Il est bien connu en hétéro-épitaxie de structures identiques, présentant un faible désaccord de maille, que les contraintes induites par ce désaccord dans le film sont en partie relaxées au-delà d'une épaisseur critique $h_{c}$, par l'apparition de 
dislocations [51]. Dans le cas étudié, cela se traduit par la transition d'une croissance 2D à une croissance 3D sur dislocations vis [52]. L'étude de la structure ci-dessus a montré que $h_{c}$ augmentait considérablement lorsque le désaccord de mailles à l'interface entre $\mathrm{YBa}_{2} \mathrm{Cu}_{3} \mathrm{O}_{7-\delta}$ et la couche sous-jacente tendait vers zéro, figure 3 , tandis que simultanément la taille des grains croissait [53].

\subsection{Les limitations}

Les limitations de la technique PLD sont d'abord l'émission de gouttelettes et le dépôt sur de grandes surfaces. En second lieu, la technique PLD est encore une association de technologies au stade expérimental qu'il est impensable de transférer en l'état en milieu industriel.

\subsubsection{L'émission de gouttelettes}

Cet effet est associé à l'instabilité du film liquide qui se forme à la surface de la cible [16]. Il est par ailleurs exacerbé par 1- la rugosité croissante de la surface, due elle-même au processus de recristallisations successives [54] et 2-différents facteurs expérimentaux, dont par exemple la porosité de la cible. Avec les caractéristiques des lasers UV utilisés aujourd'hui, tableau 2, la profondeur de propagation de l'interface solide-liquide est de l'ordre du micromètre. Dans ces conditions, il existe plusieurs processus intrinsèques à la technique PLD, qui sont susceptibles de déstabiliser la surface liquide et de projeter ainsi des gouttelettes de taille micrométrique : la force d'inertie due à la propagation de l'interface solide-liquide, la dilatation d'origine thermique de la cible et éventuellement, l'approche de l'état critique. De nombreuses solutions techniques ont été proposées pour prévenir le dépôt des gouttelettes sur le film [8], les plus satisfaisantes d'entre elles étant probablement mécaniques [55]. L'utilisation de cibles liquides, en éliminant le processus de changement de phase et en maintenant un état de surface quasi parfait, réduit considérablement la densité de gouttelettes et permet par ailleurs une simplification du dispositif de balayage de la cible par le faisceau laser $[16,56]$. Cette possibilité, utilisée par l'un des auteurs pour le dépôt de $\mathrm{HgBa}_{2} \mathrm{CaCu}_{2} \mathrm{O}_{y}$-cible de $\mathrm{Hg}$ liquide-, n'est cependant ni universelle ni exempte de défauts.

\subsection{2 le dépôt sur des grandes surfaces}

La technique PLD a jusqu'ici été un outil de laboratoire, y compris en milieu industriel. Peu d'efforts ont été consacrés à l'étude de prototypes industriels. Néanmoins, quelques résultats très prometteurs ont été récemment obtenus concernant la maîtrise des dépôts sur de grandes surfaces [57]. Les résultats les plus récents font état de dépôt sur des substrats de diamètre $15 \mathrm{~cm}$ [58], performance remarquable démontrée avec oxyde complexe, YBCO, secteur probablement le plus porteur pour les applications industrielles futures.

\subsection{Les améliorations}

Le passage de l'outil de laboratoire à un outil de production suppose au préalable que plusieurs facteurs rédhibitoires soient surmontés comme 1-l'émission de gouttelettes et 2la maîtrise de l'épaisseur et de la qualité des films sur de grandes surfaces avec une productivité acceptable et, 3 - le développement d'une technologie éprouvée. Il suppose aussi que les avantages de cette technique seront suffisamment attrayants pour supplanter les technologies déjă établies.

La suppression des gouttelettes peut être envisagée à court terme dans la continuité des actions passées. A plus long terme, une autre voie consiste à développer des lasers UV qui réduisent au minimum les risques de formation de gouttelettes, par exemple par réduction de la longueur d'onde $\lambda$ et de la durée de l'impulsion $\tau$. Cette voie est déjà abordée avec le laser $F_{2}(\lambda=157 \mathrm{~nm}, \tau=15 \mathrm{~ns})$. Elle pourrait s'appuyer sur l'activité intense aujourd'hui déployée autour des lasers UVX et à impulsion courte, $\tau \equiv \mathrm{ps,} \mathrm{fs}$ [59]. La pertinence de ces nouveaux outils pour le dépôt PLD doit au préalable être analysée des points de vue fondamental et expérimental. Les critères de productivité et 
de maîtrise de procédé ne peuvent être évalués que dans le cadre d'un développement de prototypes industriels qui prennent en compte toutes les contraintes usuelles d'un outil de production : investissement, fiabilité du procédé, opération et maintenance. Il existe dans la littérature des réponses éparses et partielles à chacun de ces critères. Le problème posé aujourd'hui, les atouts de la PLD ayant été amplement démontrés avec des équipements de laboratoire, est donc celui de la conception et de la réalisation d'un équipement PLD répondant d'abord aux critères techniques de la production, qui ne soit pas une juxtaposition de technologies (optiques, mécaniques, vide, etc...) à l'instar des équipements actuels. Ce pas franchi, il deviendrait alors possible d'apprécier l'opportunité du passage à un véritable outil de production.

\section{Conclusion}

La technique PLD est un outil, qui a déjà prouvé en laboratoire son potentiel et sa supériorité sur les techniques existantes pour le dépôt de nombreux matériaux, et notamment dans le secteur des oxycies complexes, à la base des futurs composants intégrés pour l'électronique et l'optoélectronique. Le passage à l'outil industriel dans de tels secteurs est envisageable dans la mesure où l'on aura d'abord surmonté plusieurs problèmes intrinsèques au procédé, dont l'émission de gouttelettes, et démontré sa capacité à satisfaire les critères habituels d'une exploitation industrielle. L'acquis actuel permet d'envisager avec optimisme l'avenir industriel de la technique PLD. Le prochain pas dans cette voie devrait être celui de la conception et de la réalisation d'une technologie maîtrisée, qui réponde au moins aux critères techniques de base d'un outil de production. Une telle action nécessite très probablement une forte interaction entre les équipes de recherche et les fabricants de laser et de machine de dépôt, afin de converger vers un équipement fonctionnel.

\section{Références}

[1] Smith H.M., Turner A.F., Appl.Opt. 4 (1965) 147

[2] Bednorz J.G., Müller, Z.Phys. B64 (1986) 189

[3] Wu M.K., Ashbum J.R., Torng C.J., Hor P.H., Meng R.L., Gao L., Huang Z.J., Wang Y.Q., Chu C.W., Phys. Rev. Lett 58 -9, (1987) 908

[4] Singh R.K., Narayan J., Phys. Rev. B 41 (13) (1990) 3843-8859

[5] Haglund Jr R.F., Appl. Surf. Sci. $96-98$ (1996) 1-13 ; Yilbas B.S., Sami M., Jpn. J. Appl. Phys. 34 Part 1, 12A (1995) 6391-6400

[6] Leboeeuf J.N., Chen K.R., Donato J.M., Geohegan D.B., Liu C.L., Puretzky A.A., Wood R.F., Appl. Surf. Sci. 96-98 (1996) 14-23 ; Anisimov S.I., Luk'yanchuk B.S., Luches A., Appl. Surf. Sci. 96-98 (1996) 24-32 ; Sumi H., Surf. Sci. 248 (1991) 382-410

[7] Auciello O., Ramesh R., MRS Bulletin 21-6 (1996) 31-36 ; Singh R.K., Holland O.W., Narayan

J., J.Appl. Phys. 68-1 (1990) 233-247

[8] Belouet C., Appl. Surf. Sci. 96-98 (1996) 630-642

[9] Häkkinen H., Landman U., Phys. Rev. Lett. $71-7$ (1993) 1023

[10] Geohegan D.B., Mashburn D.N., Appl. Phys. Lett. 55 (1989), 2345 ; Cheng N.H., Ying Q.Y., Sheng J.P., Kwak H.S., J.Appl. Phys. 69 (1991) 6349 ; Estler R.C., Nogar N.S., J.Appl. Phys. 69 (1991) 1654 ; Weaver I., Lewis C.L.S., J. Appl. Phys. 79-9 (1996) 7216-7222

[11] Chen C.H., Murphy T.M., Phillips R.C., Appl. Phys, Lett. 57 (1990) 937

[12] Girault C., Damiani D., Aubreton J et Catherinat A., Appl. Phys. Lett., 55 (1989) 182 et Fukishima K., Kanke Y., Morishita T., J.Appl. Phys. 74 (1993) 6948

[13] Lee.I., Callcott T.A., Arakawa E.T., Phys. Rev. B 47 (1993) 6661

[14] NoorBatcha I., Lucchese R.R., Zeiri Y., J.Chem. Phys. 86 (1987) 5816

[15] M.M. Martynyuk, Phys. Comb. and Explosion 13 (1977) 178-191 ; Kelly R., Miotello A., Appl. Surf. Sci. $96-98$ (1996) 205-215

[16] Bennett T.D., Grigoropoulos C.P., Krajnovich D.J., J.Appl. Phys.77-2 (1995) 849-864

[17] Dawson J., Kaw P., Green B., The Phys. of Fluids 12-4 (1969) 875-882 ; Tokarev V., Marine W., Lunney J.G., Sentis M., Thin Solid Films 241 (1994) 2599

[18] Deneffe K., van Miegen P., Brijs B., Vandervorst W., Mertens R., Borghs G., Jpл. J. Appl. Phys. 30, 9 A (1991) 1959

[19] Otis C.E., Dreyfus R.W., Phys. Rev. Lett. 67 (1991) 2102

[20] Yoshida T., Takeyama S., Yamada Y., Mutoh K., Appl. Phys. Lett. 68-13 (1996)1772-1774

[21] Matunari S., Kanai M., Kawai T., Jpn. J. Appl. Phys. 34 P.2, 1 A (1995) 20-22 ; Contour J.P., Sant C., Ravelosona D., Fisher B., Patlagan L., Jpn. J. Appl. Phys. 32 P.2, 8 B (1993) 1134-1136

[22] Cuomo J.J., Pappas D.L., Bruley J., Doyle J.P.,Saenger K.L., J.Appl. Phys. 70 (1991) 5629 
[23] Franks J., Enke K., Richardt A., J. Met. Mater., Inst. of Met. , Thompson D.G., Proc. 30th ann. techn. conf. SVC (1987) 143-144

[24] Enke K., Thin Solid Films 80 (1981) 227-232

[25] Kumar N., Schmidt H., Xie C., Solid State Technology. May (1995) 71

[26] Pappas D.L., Saenger K.L., Bruley J., Krakow W., Cuomo J.J., Gu T., Collins R.W., J. Appl. Phys.,71-11 (1992) 5675-5684

[27] Rist O., Murray P.T., Mater. Lett. 10, 7-8 (1991) 323

[28] Friedmann T.A., McCarty K.F., Klaus E.J., Boehme D., Clift W.M., Johnsen H.A., Mills M.J., Ottesen D.K., Appl. Phys. Lett. 61-20 (1992) 2406-2408

[29] Kools J.C.S., Nillesen C.J.C., Bromgersma S.H., van de Riet E., Dieleman J., J. Vac. Sci. Technol. A 10-4 (1992) 1809-1814

[30] Nashimoto K., Fork D.K., Ponce F.A., Tramontana J.C., Jpn. J. Appl. Phys. 32 P.1, 9 B (1993) 4099-4102

[31] Ghonge S.G., Goo E., Ramesh R., Appl. Phys. Lett. 62-15 (1993) 1742-1744

[32] Tabata H., Murata O., Kawai T., Kawai S., Okuyama M., Jpn. J. Appl. Phys. 32 P.1, 12A (1993) $5611-5614$

[33] Wu N.J., Ignatiev A., Mesarwi A.W., Lin H., Xie K., Shih H.D., Jpn. J. Appl. Phys. 32 P.1, 11 A (1993) 5019-5023 ; Bhattacharya P., Komeda T., Park K., Nishioka Y., Jpn. J.Appl. Phys. 32 P.1, 9B (1993) 4103-4106

[34] Kidoh H., Ogawa T., Yashima H., Morimoto A., Shimizu T., Jpn. J. Appl. Phys. 31 P.1, 9 B (1992) 2965-2967 ; Nakamura T., Yamanaka Y., Morimoto A., Shimizu T., Jpn. J.Appl. Phys. 34 P.1, 9B (1995) 5150

[35] Grosse-Holz K.O., Cillessen J.F.M., Waser R., Appl. Surf. Sci. 96.98 (1996) 784-790

[36] Simion B.M., Thomas G., Ramesh R., Keramidas V.G., Pfeffer R.L., Appl. Phys. Lett. 66-7 (1995) 830-832

[37] Lecoeur P., Gupta A., Ducombe P.R., Gong G.Q., Xiao G., J.Appl. Phys. 60-1 (1996) 513-517

[38] Shibata Y., Kuze N., Matsui M., Kanno Y., Kaya K., Ozaki M., Kanai M., Kawai T., Jpn.J.Appl. Phys. 34 P.1, 1(1995) 249

[39] Nagaishi T., Itozaki H., J.Vac. Sci. Technol. A 14-4 (1996) 1995 ; Trotel A., Pyee M., Lavigne B., Chambonnet D., Lederer P., Appl. Phys. Lett. 68-18 (1996) 2559-2561

[40] Miyashita S., Higuma H., Uchikawa F., Jpn. J. Appl. Phys. 33 P.2, 7 A (1994) 931

[41] Kools J.C.S., Coehoorn R., Hakkens F.J.G., Fastenau R.H.J., J.Magn. Magn. Mater. 121(1993) 83-87

[42] Geurtsen A.J.M., Kools J.C.S., de Wit L., Lodder J.C.. Appl. Surf. Sci. 96-98(1996) 887-890

[43] Singh R.K., Bhattacharya D., Sharan S., Tiwari P., Narayan J., J. Mat. Res. 7 (1992)2639-2641

[44] van Maaren A.P.J., Sinke W.C., Flicstein J., Appl. Phys. Lett. 62-5 (1993) 479

[45] Blanchet G.B., Shah S.I., Appl. Phys. Lett. 62-9 (1993) 1026-1028

[46] Ohtsuka S., Koyama T., Tsunetomo K., Nagata H.; Tanaka S., Appl. Phys. Lett. 61-25 (1992) 2953-2954

[47] Katz A., Lee C.H., Tai K.L., Mater. Chem. Phys., 37 (1994) 303-328

[48] Geohegan D.B., Puretzky A.A., Appl. Phys. Lett. 67-2 (1995) 197-199

[49] AMI : superalliage monocristallin à base nickel (SNECMA); DeMasi-Marcin J.T., Gupta D.K., Surf. Coating. Technol. 68/69 (1994) 1-9

[50] Chambonnet D., Mehri F., Degoy S., Lederer P., Belouet C., Fages C., Keller D., Carru J.C., Correra L., à paraître dans : Physica C 271-182 (1996)

[51] van der Merwe J.H., J.Appl. phys. 34 (1963) 117

[52] Keller D., Gervais A., Chambonnet D., Belouet C., Audry C., J.Phys. Inl France 5 (1995) 135-143

[53] Keller D., Chambonnet D., Belouet C., à paraître.

[54] Spindler H.L., Gilgenbach R.M., Lash J.S., App], Phys. Lett. 68-23 (1996) 3245-3247

[55] Pechen E.V., Varlashkin A.V., Krasnosvobodtsev. S.I., Brunner B., Renk K.F., Appl. Phys. Lett. 66-17 (1995) 2292-2294

[56] Sankur H., Gunning W.J., DeNatale J., Flintoff J., Appl. Phys. Lett. 68 (1989) 2475 ; Rong F., Appl. Phys. Lett. 67-7 (1995) 1022-1024

[57] Panzner M., Dietsch R., Holz Th., Mai H., Völlmar. S., Appl. Surf. Sci. 96-98 (1996) 643-648; Lorenz M., Hochmuth H., Natusch D., Börner H., Lippold G., Kreher K., Schmitz W., Appl. Phys. Lett., 68-23 (1996) 3332-3334 ; Fernandez F.E., J.Vac. Sci. Technol. 13-2 (1995) 421-427

[58] Greer J.A., Tabat M.D., J. Vac. Sci. Technol. A13 (1995) 1175

[59] ce volume ; Museur L., Zheng W.Q., Kanaev A.V., Castex M.C., IEEE J. of selected topics in Quantum Electron. 1-3 (1995) 900-907 